\title{
A Novel ACTL6A Exonic Circular RNA Exacerbates Polycystic Ovary Syndrome via Recruiting TET1
}

\author{
Lingzhi Zhang \\ Cao County People's Hospital \\ Fang Wang \\ Cao County People's Hospital \\ Dongmei Li \\ Cao County People's Hospital \\ Yufeng Yan \\ Cao County People's \\ Hongyan Wang ( $\nabla$ Wang_HongY@outlook.com ) \\ Cao County Peoples Hospital
}

\section{Research}

Keywords: polycystic ovary syndrome, circRNA, DNA methylation, human granulosa-like tumor cell line (KGN) cells

Posted Date: February 24th, 2021

DOl: https://doi.org/10.21203/rs.3.rs-231266/v1

License: (c) (i) This work is licensed under a Creative Commons Attribution 4.0 International License.

Read Full License 


\section{Abstract}

Polycystic ovary syndrome (PCOS) is an endocrine disorder prevailing among females at reproductive age. Circular non-coding RNAs (circRNAs) are essential "player" in PCOS. This study aimed to investigate the potentials of newly found circRNA, circ_ACTL6A in PCOS. Dehydroepiandrosterone (DHEA) was utilized to establish PCOS model in vivo. qRT-PCR and western blot were applied to determine the gene expression level. DNA methylation was determined by bisulfite sequencing assay. RNA pull-down, Chromatin immunoprecipitation (ChIP) assay, and RNA immunoprecipitation (RIP) assay was applied to verify gene interactions. CCK-8, colony formation and 5-Ethynyl-2'-deoxyuridine (EdU) assay were applied to determine cellular function of human granulosa-like tumor cell line (KGN) cells. The results showed that circ_ACTL6A was upregulated in PCOS, which was due to the upregulation of RNA splicing factor eukaryotic translation initiation factor 4A3 (EIF4A3) and its cognate protein actin like 6A (ACTL6A). Downregulation of circ_ACTL6A suppressed the cell viability, colony formation and proliferation of KGN cells in vitro. Moreover, knockdown of circ_ACTL6A suppressed the progression of PCOS, manifested by the improvement in insulin sensitivity, ovarian function and fertility. Furthermore, circ_ACTL6A recruited tet methylcytosine dioxygenase 1 (TET1) to suppress DNA methylation of ACTL6A. Overexpression of ACTL6A promoted the development of PCOS in vivo and in vitro. Taken together, circ_ACTL6A/ACTL6A may form a positive feedback in the progression of PCOS. This may be a potential therapeutic target for PCOS.

\section{Introduction}

Polycystic ovary syndrome (PCOS) is the most common endocrinopathy and affecting $6-10 \%$ of females of reproductive age [1]. The main syndromes of PCOS are hyperandrogenism, ovulatory dysfunction, and polycystic ovaries [2]. Moreover, this metabolic disorders is associated with cardiovascular diseases, type 2 diabetes mellitus, and infertility. Nonetheless, the pathogenesis of PCOS is still unclear. Dewailly et al evidence that PCOS is the results the alteration of folliculogenesis, which is characterized by follicle maturation, proliferation, differentiation, and apoptosis [3]. Granulosa cells, surrounding follicles, provide nutrients and growth factors for oocyte differentiation and proliferation [4]. However, dysfunction of granulosa cells contributes to aberrant folliculogenesis [5]. However, the underlying molecular mechanism is still unclear.

Circular RNAs (circRNAs) originated from precursor mRNA (pre-mRNA) via head-to-tail backsplicing, are characterized by covalently closed loop and lacking 5' cap and 3' poly A tail [6]. This confer its stability in various biological functions, such as post-transcription, RNA-related protein binding, gene splicing, and modification of parental gene expression [7, 8]. However, dysfunction of circRNAs is evidenced to be associated with endocrinopathy, such as type 2 diabetes mellitus, cardiovascular diseases, obesity, and PCOS [9-12]. For instance, knockdown of circRNA_DMNT3B contributes to diabetic nephropathy via regulating miR-20b-5p/BAMBI [13]. Loss of circ_Nfix enhances cardiac regenerative repair and restores heart function via promoting the interaction between Y-box binding protein 1 and Nedd4I and sponging miR-214 to regulate glycogen synthase kinase $3 \beta$ [14]. Liu et al reveal that circRNAs, such as, 
circRNA_006555, circRNA_012588, and circRNA_005578, interacts with its parental genes to participates in age-related macular degeneration in vivo [15]. Decreased exosomal circ_LDLR suppresses estradiol production in PCOS via sponging miR-1294 [16]. circ_PUM1 suppresses the proliferation and promotes the apoptosis of granulosa cell via sponging miR-760 [17]. circ_ACTL6A is located in Chromosome 3, the fragment transfer of which genetically modified the characterization of the 3p12.3-pcen region in ovarian cancer [18]. However, the potential roles of circ_in PCOS have not been elucidated.

The chromatin is modulated by the following mayor epigenetic mechanisms: DNA methylation on the cytosine residues of $\mathrm{CpG}$ dinucleotides; covalent modifications of histone tails; and ATP-dependent chromatin remodeling [19]. However, loss of a ATP-dependent ACTL6A chromatin remodeling complexes, BRG1/BRM-associated factors (BAF) may alter DNA methylation via recruiting DNA methylases or regulating DNA-methylation related protein [20]. The complexes alter gene levels via opening chromatin and exchanging nucleosomes. This is looming ahead of human endocrinopathy following the evidence which indicates BAF subunit mutations in the tumorigenesis of prostate cancer, lipogenesis, Coffin-Siris syndrome, and hyperandrogenism [21-24]. These evidence reveals that aberrant level of BAF complexes makes rate-limiting contributions to the establishment of the diversity, stability and plasticity found in endocrine system. However, the potential role of BAF in PCOS unclear.

\section{Materials And Methods Study approval}

This study was approved by the Ethics Committee of Cao County Peoples Hospital. The relevant animal experiments was performed in accordance with the principles of Animal Care Board of Cao County Peoples Hospital.

\section{Clinical specimens}

Clinical specimens were collected from volunteers (26-33 years old) at Cao County Peoples Hospital from April 1st, 2018 to Jun 1st, 2020. All the participants had not receive any hormonotherapy at least 3 months prior to this study. PCOS patients were diagnosed in reference to the revised Rotterdam criteria. Healthy control were the volunteers with Non-PCOS women with regular menstrual cycles and normal ovarian morphology were identified as the control group.

\section{Cell culture}

Cells was established using cells from a PCOS patient as previously described [25]. KGN cells were incubated in DMEM medium supplemented with $12 \% \mathrm{FBS}$ at $37^{\circ} \mathrm{C}$ in $5 \% \mathrm{CO}_{2}$.

\section{Transfection}

Short hairpin circ_ACTL6A (shcirc_ACTL6A), circ_ACTL6A overexpression plasmids (circ_ACTL6A OE) pcDNA3.1-ACTL6A, and the negative control (NC) were obtained from GenePharma, Shanghai. Cells were transfected with shcirc_ACTL6A or sh-NC, pcDNA3.1-ACTL6A and pcDNA3.1 using Liposome 2000 
according to the manufacturer's instructions for $48 \mathrm{~h}$. After transfection, cells were used in the following experiment.

\section{Adenoviral and lentiviral vector construction}

Adenoviruses expressing shcirc_ACTL6A, lentiviruses expressing ACTL6A, and its negative control were purchased from GenePharma, Shanghai.

\section{CCK-8 assay}

Cell viability was detected with a CCK-8 kit (GIpBio, USA). Briefly, the transfected cells were lysed and washed with PBS thrice. Then cells were plated into a 96-well plate ( $2 \times 10^{3}$ cells/plate) and cultured for 96 h. At 24, 48, 72 and 96 h, the cells were incubated with $10 \mu$ of CCK-8 solutions for $2 \mathrm{~h}$. Subsequently, cell viability was determined using a microplate reader (Molecular Devices, USA).

\section{Colony formation assay}

After $48 \mathrm{~h}$ transfection, cells were seeded in a 96 -well plate $\left(2 \times 10^{3}\right.$ cells/well) and incubated at $37^{\circ} \mathrm{C}$ in $5 \% \mathrm{CO}_{2}$ for 14 days. Then cells were fixed with $4 \%$ ethanol and stained with $1 \%$ crystal violet. The stained cells were visualized with inverted light microscope (Olympus, Japan) and counted.

\section{EdU assay}

Cells were seeded into a 96 -well plate $\left(3 \times 10^{3}\right.$ cells/well). Cells were added with $50 \mu \mathrm{M}$ of EdU solutions. Then cells were washed with PBS for three times and premeated with $0.5 \%$ Triton X-100. Afterwards, cells added with $100 \mathrm{ml}$ of Hoechst 33342 and incubated at room temperature for $30 \mathrm{~min}$. The results were captured by fluorescent microscope (Nikon, Japan).

\section{RNA pull-down assay}

Biotin-labeled RNAs were transcribed in vitro with Biotin RNA Labeling Mix and T7 RNA polymerase (Roche, Switzerland). $5 \mu \mathrm{g}$ RNA mixed with cell extract and incubated for for $1 \mathrm{~h}$ at $4^{\circ} \mathrm{C}$. After washed with PBS thrice, RNA-protein binding mixture was boiled. The samples were determined using western blot or mass spectrometry.

\section{RNA immunoprecipitation (RIP)}

KGN cells were lysed by RIP lysis and incubated with RIPA buffer containing magnetic beads and antiAgo2. IgG served as loading control. RNA was purified using proteinase K. RNA quality and quantity was detected. Finally, RNA purification was performed using qRT-PCR.

\section{Chromatin immunoprecipitation (ChIP) assay}

ChIP assay was performed using a EZ-ChIP kit (Millipore, USA). After 48-hour transfection, cells were fixed with $1 \%$ formaldehyde and sonicated on ice. Then cells were incubated with anti-TET1 antibody and normal rabbit lgG. Precipitated DNA was analyzed by qRT-PCR.

\section{DNA methylation}


Total DNA were extracted from KGN cells using a Genomic DNA Purification Kit (GeneJET, USA). After treated with bisulfite, DNA was amplified using PCR. PCR products were gel purified and directly ligated. The status of DNA methylation was detected using restriction enzyme digestion as previously described [26]

\section{qRT-PCR}

Total RNA was collected from cells using TRIzol reagent (Invitrogen). RNA was reversely transcribed into cDNA by a PrimeScript RT Reagent kit (Takara, Japan). PCR was conducted using SYBR Green PCR kits (Takara, Japan) on a 7000 Sequence Detection System (Applied Biosystems, USA). The thermocycling conditions were as followed: initial activation at $50^{\circ} \mathrm{C}$ for $2 \mathrm{~min}$, and denaturation at $95^{\circ} \mathrm{C}$ for $10 \mathrm{~min}$, followed by 40 cycles of $95^{\circ} \mathrm{C}$ for $15 \mathrm{sec}$ and $60^{\circ} \mathrm{C}$ for $1 \mathrm{~min}$.. Finally, the expression levels were calculated with $2^{-\triangle \triangle C t}$ method. GAPDH served as loading control. The sequences of the primers used were as followed: circ_ACTL6A, F: 5'-ATCCCCTCTTGCTGGAGACT-3' and R: 5'-

TGTGCCAAGACCTCGTAACC-3'; 5'-CCAGGTCTCTATGGCAGTGTAA-3' and reverse, 5'CGTAAGGTGACAAAAGGAAGGTA-3'; EIF4A3, 5'-AAGGGAGAGATGTCATCGCAC-3' and R: 5'GCTTGAGTTTCACGAACCTGA-3' GAPDH, F: 5'-GGAGCGAGATCCCTCCAAAAT-3' and R: 5'GGCTGTTGTCATACTTCTCATGG-3'.

\section{Western blot}

Total protein was isolated from cells. The protein concentration was determined by a BCA kit (Abcam, USA). Equal amount of protein $(30 \mu \mathrm{g})$ was separated by $12 \%$ SDS-PAGE. The protein was transferred onto PVDF membranes. The membranes were incubated with primary antibodies including anti-ACTL6A (ab189315, 1: 1000, Abcam, Shanghai), anti-EIF4A3 (ab180573, 1: 1000, Shanghai, USA), anti-TET1 (ab272900, 1: 1000, Abcam, Shanghai), GAPDH (ab9485, 1: 1000, Abcam, Shanghai) at room temperature for $2 \mathrm{~h}$. Afterwards, the membranes were incubated with secondary antibodies (ab6721, 1: 5000, Abcam, USA). Subsequently, the bands were visualized using a ECL kit (Beyotime, Shanghai) and analyzed on ImageJ software $(\mathrm{NIH})$.

\section{Animal models}

60 female C57BL/ 6 mice (21 days old, 18 22g) were purchased Animal Center of Nanjing Medical University (Nanjing, China). Mice were randomly divided 4 groups, and kept in a controlled condition (20 \pm $2^{\circ} \mathrm{C}$ ) with a $12 \mathrm{~h}$ light/ $12 \mathrm{~h}$ dark cycle, free access to food and water. The mice in control group were injected daily with sesame oil $(0.1 \mathrm{~mL})$. The PCOS model was established by injecting dehydroepiandrosterone (DHEA, $6 \mathrm{mg} / 100 \mathrm{~g}$ body weight) dissolved in sesame oil daily for 3 weeks. The PCOS mice were randomly divided into ad-shcirc_ACTL6A, adsh-NC, LV-ACTL6A, and LV-NC group. After 3 weeks, glucose tolerance test (GTT) and insulin tolerance test (ITT) were applied to detect fasting blood glucose and fasting insulin were detected and calculated according to homeostasis model assessment of insulin resistance (HOMA-IR) method. Two weeks later, the mice were sacrificed. 


\section{GTT and ITT.}

Mice were fasted for GTT and ITT experiments. The glucose level was detected by a blood glucose AccuChek Performa (Roche Diagnostics). Then mice were injected with d-glucose or insulin. Subsequently, the levels of glucose and insulin were 15, 30, 60, 90 and 120 min.

\section{Histological analysis}

Ovarian and uterine tissues were fixed in 4\% paraformaldehyde, embedded in paraffin, and sliced into 5 $\mu \mathrm{m}$ section. Afterwards, the section was stained with haematoxylin-eosin (HE) and visualized by ight microscope (Leica Microsystems, Germany).

\section{Statistical analysis}

The data were analyzed using SPSS 19.0 and represented as mean \pm SD. The difference in groups were analyzed by one-way ANOVA assay followed by Newman-Keuls test. $\mathrm{P}<0.05$ was deemed significantly different.

\section{Results}

\section{Clinical features}

Compared with healthy control, the levels of body mass index (BMI), luteinizing hormone (LH), basal testosterone $(\mathrm{T})$, anti-Müllerian hormone (AMH), fasting insulin, and HOMA-IR were significantly increased in PCOS patients, while basal follicle-stimulating hormone (FSH) was significantly decreased. However, there was no significant difference in age.

\section{circ_ACTL6A is overexpressed in PCOS}

To explored the potential roles of circRNAs in PCOS. We investigated the expression of circRNA in PCOS patients (GSE145296). As showed in Fig. 1A and B, the expression of circ_0122825 (circ_ACTL6A) was overexpressed in PCOS patients. To further verify the roles of circRNA in PCOS. We also determined the levels of circ_ACTL6A in PCOS patients and KGN cells. The results showed that circ_ACTL6A was overexpressed in PCOS patients ad KGN cells (Fig. 1C and D). Divergent primers spanning the backsplicing sites were utilized to determine the level of circ_ACTL6A (Fig. 1E). The results from circPrimer (v.1.2.0.5) showed that circ_ACTL6A is produced by the back splicing of pre-ACTL6A mRNA exons $4 \sim 8$ (Fig. 1F). Divergent and convergent primers was designed to amplify circ_ACTL6A and linear ACTL6A, respectively. Then we found that circ_ACTL6A was only amplified in CDNA (Fig. 1G). Moreover, circ_ACTL6A due to its circular properties, was more stable than mACTL6A after in cells exposed to actinomycin D (Fig. 1H). Additionally, circ_ACTL6A was strictly resistant to RNase R digestion (Fig. 1I). Collectively, theses results verified the circularity of circ_ACTL6A.

\section{Knockdown of circ_ACTL6A suppressed the proliferation, migration, and invasion of KGN cells}


We analyzed circRNA-miRNA interaction in relation to regulatory RNA motifs and elements via the online database Circinteractome (https://circinteractome.nia.nih.gov/circular_rna.html) and and GO analysis (mirPath v.3; http://snf-515788.vm.okeanos.grnet.gr) (Supplementaty materials). The results showed that circ_ACTL6A regulated cell behaviors, DNA modification, and insulin secretion (Fig. 2A). To further investigated the roles of circ_ACTL6A in PCOS, we downregulated it though silencing its expression in KGN cells using short hairpin RNA. The results showed that the expression of circ_ACTL6A was significantly decreased in cells transfected with sh-circ_ACTL6A, especially in sh-circ_ACTL6A 2\# group (Fig. 2B). Downregulated circ_ACTL6A significantly suppressed cell viability of KGN cells (Fig. 2C). Moreover, knockdown of circ_ACTL6A remarkably decreased the colony number of KGN cells (Fig. 2D and E). The proliferation of KGN cells was significantly inhibited by sh-circ_ACTL6A (Fig. 2F and G).

\section{Knockdown of circ_ACTL6A improved insulin resistance, ovarian dysfunction and infertility in mice with PCOS}

To further identify the potential roles of circ_ACTL6A in PCOS, we further investigated the possible effects of circ_ACTL6A knockdown on the progression of PCOS in vivo via injecting mice with ad-shcirc_ACTL6A. The results from glucose tolerance tests (GTTs), insulin tolerance tests (ITTs) showed that knockdown of circ_ACTL6A significantly decreased insulin resistance in comparison with DHEA group (Fig. 3A and B). Furthermore, knockdown of circ_ACTL6A alleviated disrupted estrous cycle (Fig. 3C and D). The histological assay showed that ovaries were composed of follicles at different stages and dictated regular numbers of corpora lutea. However, DHEA injection contributed to the increase of cyst-like follicles and decrease of corpora lutea, which was reversed by circ_ACTL6A knockdown (Fig. 3E). Additionally, downregulation of circ_ACTL6A improved morphological changes of mice ovary (Fig. 3F-I). Furthermore, we investigated the potential effects of circ_ACTL6A knockdown on mice fertility after mating. As showed in Fig. $3 \mathrm{~J}$, the decrease of pups induced by DHEA was reversed by circ_ACTL6A knockdown. These data suggested that knockdown of circ_ACTL6A alleviated insulin resistance and infertility in mice with PCOS.

\section{EIF4A3 induces the upregulation of circ_ACTL6A}

The RNA splicing factor is the crucial factor for the expression of circRNAs. RAP assay was applied to determine the protein binding to pre-ACTL6A. We first determined the mRNA leve of ACTL6A in KGN cells. As showed in Fig. 4A, the expression of ACTL6A was significantly increased in KGN cells. The RNA The results of RNA pull-down assay and mass spectrometry analysis showed that the splicing factor EIF4A3 (37 kd) interacted with pre-ACTL6A (Fig. 4B). The expression of EIF4A3 was overexpressed in KGN (Fig. 4C-E). RIP assay showed that interacted with circ_ACTL6A (Fig. 4F). The expression of EIF4A3 was significantly decreased in cells transfected with shEIF4A3, which was more potent in shEIF4A3 1\# group (Fig. 4G) Knockdown of EIF4A3 supressed the expression of circ_ACTL6A (Fig. 4H).

\section{circ_ACTL6A upregulates ACTL6A}

As circ_ACTL6A binds to the ACTL6A promoter, we hypothesized that circ_ACTL6A may interact with ACTL6A to promote the progression of PCOS. As showed in Fig. 5A-C, knockdown of circ_ACTL6A significantly suppressed the expression of ACTL6A both in mRNA and protein level. 


\section{circ_ACTL6A induces CpG DNA demethylation in the ACTL6A promoter}

We explored the possible epigenetic mechanisms that circ_ACTL6A increased the expression of ACTL6A. The binding sequences of circ_ACTL6A to ACTL6A was predicted by Softberry (http://www.softberry.com/berry.phtml?topic=cpgfinder\&group=programs\&subgroup=promoter). The conditions were: Min length of island to find, 200; Min percent G and C, 50; Min gc_ratio = $P(C p G) /($ expected) $P(C p G), 0.600$. Then we found that there was only one $\mathrm{CpG}$ islands in the proximal promoter of ACTL6A. This study focused on the methylation status of the CpG islands in the proximal promoter of ACTL6A (Fig. 6A). The sodium bisulfite sequencing results showed that circ_ACTL6A induced extensive DNA demethylation in comparison with vector group (Fig. 6B). These results suggested that extensive DNA demethylation in the promoter of promoter of circ_ACTL6A was associated with the upregulation of circ_ACTL6A positive cells.

\section{circ_ACTL6A recruits TET1}

TET1 is a key regulator of female germ cell development and zygote genome reprogramming via modulating DNA demethylation. To verify whether the binding of circ_ACTL6A to ACTL6A could promote KGN cell proliferation and zygote genome reprogramming via regulating TET1, RIP assay was performed to validate the interaction between circ_ACTL6A and TET1 (Fig. 7A-D).

\section{Overexpressed ACTL6A reverses the effects of circ_ACTL6A knockdown on the proliferation of KGN in vitro}

Rescue assays were applied to verify the interaction between circ_ACTL6A and ACTL6A in PCOS. As showed in Fig. 8A, the expression of ACTL6A was significantly increased in ACTL6A OE group, suggesting that cells were successfully transfected. Overexpressed ACTL6A abated the effects of circ_ACTL6A knockdown and increased the cell viability, colony formation, and proliferation of KGN cells (Fig. 8B-F).

\section{Discussion}

Augmenting evidence have certificated the potentials of circRNAs and the underlying molecular mechanisms [6-8]. circRNAs collectively participate in the initiation and development of endocrine dyscrasia in females. For instance, dysregulated circRNAs are associated with the progression of gestational diabetes mellitus, cardiovascular diseases, obesity, and PCOS [9-12]. Overexpressed circRNA_0054633 contributes to gestational diabetes mellitus and the increase of glycosylation index [27]. Knockdown of hsa_circ_0118530 suppresses oxidative stress, inflammation, and human granulosalike tumor cell injury [28]. Upregulation of circ_ASPH, circ_LDLR, and circPUM1 promote the proliferation, migration and invasion of KGN cells, which is a crucial factor for PCOS [16, 17, 29]. circRNAs function as competitive RNAs during the initiation and progression of PCOS. However, the knowledge of circRNA in PCOS is limited. 
In the present study, circ_ACTL6A was overexpressed in PCOS in vivo and in vitro. The results of clinical data dictated that upregulation of circ_ACTL6A was closely associated with the progression of PCOS. To verify the roles of circ_ACTL6A in PCOS, GO and KEGG analysis indicated that circ_ACTL6A regulated insulin secretion. cellular functions, such as cell motility and proliferation. In vivo assay showed that knockdown of circ_ACTL6A decreased cystic follicle and suppressed insulin resistance, which is a crucial factor for PCOS. Moreover, knockdown of circ_ACTL6A improved ovarian dysfunction and infertility. The progression of PCOS is characterized by proliferative ability of KGN cells. In this study, knockdown of circ_ACTL6A suppressed the proliferation of KGN cells. However, the various functions of circRNAs are largely associated with its specific structure. More studies are still needed to explore the specific structure and potential functions in PCOS.

Most circRNAs are formed by back-splicing of precursor mRNAs [6-8]. However, the expression of circRNA should carefully be differentiated from the effects of the changes of its cognate protein in that most circRNAs are alternative splicing products from protein-coding genes [30]. ACTL6A, a subunit gene of BAF complex, encodes a family member of actin-related proteins (ARPs), which are crucial regulator of vesicular transport, nuclear migration and chromatin remodeling. ACTL6A functions as activator of epithelial-mesenchymal transition (EMT) to induce exacerbate hepatocellular carcinoma (HCC) and a chromatin-remodeling factors to modulate biological processes including cell proliferation and differentiation via repressing SWI/SNF-dependent induction of KLF4 [31, 32]. Moreover, overexpression of ACTL6A enhances follicle-stimulating hormone induced glycolysis in ovary cancer via activating PGK1, which is the hallmark of cancer [33]. These suggest that ACTL6A may regulates the levels of genes involved in the EMT, proliferation, differentiation, and DNA modification. Thence, we hypothesized ACTL6A may participate in the progression of PCOS via regulating its cognate protein ACTL6A. circ_ACTL6A, a transcriptional byproduct of ACTL6A, modulated the motility of KGN cells at transcription level. Moreover, splicing factors (SFs) are deeply involve in regulating the trasncription of precursor mRNA and synthesis of circRNAs [34, 35]. We, thereafter, investigated the potential roles of SFs in the upregulation of PCOS. We found that a SF EIF4A3 induced the upregulation of circ_ACTL6A and ACTL6A. These results suggested that precursor mRNA splicing factors participated in the upregulation of circ_ACTL6A in PCOS.

Bioinformatics analysis showed that circ_ACTL6A could function as ceRNA to modulate genes related to chromatin mortification and DNA-templated processes. circ_ACTL6A bound to its cognate protein in cis and induced the demethylation. Previous studies evidence that circRNAs modulate DNA methylation via recruiting DNA methylases or regulating DNA-methylation related protein [36]. TET1, as a member of Teneleven translocation enzymes, oxidize $5 \mathrm{mC}$ into 5 -hydroxymethylcytosine $(5 \mathrm{hmC})$, which activates demethylation and modulates gene expression [37]. In this study, circ_ACTL6A recruited TET1 to form a positive feedback loop to induce DNA hypomethylation, which promoted the transcription of ACTL6A.

Furthermore, some circRNAs are associated with the translation of ribosomes [38]. The accumulation of circ_ERBB2 in nucleoli modulates ribosomal DNA transcription and suppresses cellular proliferation [39]. Generally, these ribo-circRNAs utilizes the start codon of its hosting mRNA. Sequences analysis showed 
that circ_ACTL6A contained the start codon ATG of ACTL6A. Positively translated circ_ACTL6A induced abridged ACTL6A. On the other hand, ACTL6A may further modulate ribosome synthesis. The roles of circRNAs in ribosomal DNA transcription in PCOS are needed for further study.

\section{Conclusion}

In conclusion, circ_ACTL6A was a alternative product of ACTL6A. The circRNA was overexpressed in PCOS. circ_ACTL6A bound to the promoter of ACTL6A to upregulate its cognate protein. Moreover, circ_ACTL6A recruited TET1 to induce DNA hypomethylation of ACTL6A. This formed a positive feedback in PCOS. Thence, the ACTL6A circRNA can be a promising biomarker for PCOS.

\section{Abbreviations}

PCOS polycystic ovary syndrome

circRNA circular non-coding RNA

ChIP chromatin immunoprecipitation

RIP RNA immunoprecipitation

EdU 5-Ethynyl-2'-deoxyuridine

KGN cells human granulosa-like tumor cell line cells

ACTL6A actin like $6 \mathrm{~A}$

EIF4A3 eukaryotic translation initiation factor $4 \mathrm{A3}$

TET1 tet methylcytosine dioxygenase 1

BAF BRG1/BRM-associated factors

GTT glucose tolerance test

ITT insulin tolerance test

HOMA-IR homeostasis model assessment of insulin resistance

HE haematoxylin-eosin

BMI body mass index

LH luteinizing hormone

T testosterone 
$\mathrm{AMH}$ anti-Müllerian hormone

FSH follicle-stimulating hormone

ARPs actin-related proteins

EMT epithelial-mesenchymal transition

\section{Declarations}

\section{Ethics approval and consent to participate}

This study was approved by the Ethics Committee of Cao County Peoples Hospital and Animal Care Board of Cao County Peoples Hospital. All the volunteers enrolled in this study provided informed consent.

\section{Consent for publication}

All the volunteers were consent to this publication.

\section{Availability of data and materials}

All data generated or analyzed during this study are included in this published article.

\section{Competing interest}

No.

\section{Funding}

None.

\section{Authors' contributions}

LZ drafted this work; and cooperated with FW and DL to do the experiment data and collected the data; and with $Y Y$ to interpret the data. HW designed and revised this paper.

\section{Acknowledgements}

Not applicable.

\section{References}

1. Risal S, Pei Y, Lu H, Manti M, Fornes R, Pui HP, Zhao Z, Massart J, Ohlsson C, Lindgren E, et al. Prenatal androgen exposure and transgenerational susceptibility to polycystic ovary syndrome. Nat Med. 2019;25(12):1894-1904. 
2. McCartney CR, Marshall JC. CLINICAL PRACTICE. Polycystic Ovary Syndrome. N Engl J Med. 2016;375(1):54-64.

3. Dewailly D, Robin G, Peigne M, Decanter C, Pigny P, Catteau-Jonard S. Interactions between androgens, $\mathrm{FSH}$, anti-Mullerian hormone and estradiol during folliculogenesis in the human normal and polycystic ovary. Hum Reprod Update. 2016;22(6):709-724.

4. Dewailly D, Robin G, Peigne M, Decanter C, Pigny P, Catteau-Jonard S. Interactions between androgens, $\mathrm{FSH}$, anti-Mullerian hormone and estradiol during folliculogenesis in the human normal and polycystic ovary. Hum Reprod Update. 2016;22(6):709-724.

5. Hsueh AJ, Kawamura K, Cheng Y, Fauser BC. Intraovarian control of early folliculogenesis. Endocr Rev. 2015;36(1):1-24.

6. Li X, Yang L, Chen LL. Mol Cell. The Biogenesis, Functions, and Challenges of Circular RNAs. 2018 Aug 2;71(3):428-442.

7. Zhang Y, Xue W, Li X, Zhang J, Chen S, Zhang JL, Yang L, Chen LL. The Biogenesis of Nascent Circular RNAs. Cell Rep. 2016;15(3):611-624.

8. Chen LL. The biogenesis and emerging roles of circular RNAs. Nat Rev Mol Cell Biol. 2016;17(4):20511.

9. Kameswaran V, Golson ML, Ramos-Rodríguez M, Ou K, Wang YJ, Zhang J, Pasquali L, Kaestner KH. The dysregulation of the DLK1-MEG3 locus in islets from patients with Type 2 diabetes is mimicked by targeted epimutation of its promoter with TALE-DNMT constructs. Diabetes. 2018;67(9):18071815.

10. Aufiero S, Reckman YJ, Pinto YM, Creemers EE. Circular RNAs open a new chapter in cardiovascular biology. Nat Rev Cardiol. 2019;16(8):503-514.

11. Liu Y, Liu H, Li Y, Mao R, Yang H, Zhang Y, Zhang Y, Guo P, Zhan D, Zhang T. Circular RNA SAMD4A controls adipogenesis in obesity through the miR-138-5p/EZH2 axis. Theranostics. 2020;10(10):4705-4719.

12. Liu J, Ding J, Qu B, Liu J, Song X, Suo Q, Zhou A, Yang J. CircPSMC3 alleviates the symptoms of PCOS by sponging miR-296-3p and regulating PTEN expression. J Cell Mol Med. 2020;24(18):1100111011.

13. Zhu K, Hu X, Chen H, Li F, Yin N, Liu AL, Shan K, Qin YW, Huang X, Chang Q, et al. Downregulation of circRNA DMNT3B contributes to diabetic retinal vascular dysfunction through targeting miR-20b-5p and BAMBI. EBioMedicine. 2019;49:341-353.

14. Huang S, Li X, Zheng H, Si X, Li B, Wei G, Li C, Chen Y, Chen Y, Liao W, et al. Loss of Super-EnhancerRegulated circRNA Nfix Induces Cardiac Regeneration After Myocardial Infarction in Adult Mice. Circulation. 2019;139(25):2857-2876.

15. Liu X, Zhang L, Wang JH, Zeng H, Zou J, Tan W, Zhao H, He Y, Shi J, Yoshida S, et al. Investigation of circRNA Expression Profiles and Analysis of circRNA-miRNA-mRNA Networks in an Animal (Mouse) Model of Age-Related Macular Degeneration. Curr Eye Res. 2020;45(9):1173-1180. 
16. Huang X, Wu B, Chen M, Hong L, Kong P, Wei Z, Teng X. Depletion of exosomal circLDLR in follicle fluid derepresses miR-1294 function and inhibits estradiol production via CYP19A1 in polycystic ovary syndrome. Aging (Albany NY). 2020;12(15):15414-15435.

17. Deng $L$, Chen $Q$, Xie J, Wei W, Hui H. circPUM1 promotes polycystic ovary syndrome progression by sponging to miR-760. Gene. 2020 Sep 5;754:144903.

18. Cody NA, Shen Z, Ripeau JS, Provencher DM, Mes-Masson AM, Chevrette M, Tonin PN. Characterization of the 3p12.3-pcen region associated with tumor suppression in a novel ovarian cancer cell line model genetically modified by chromosome 3 fragment transfer. Mol Carcinog. 2009;48(12):1077-92.

19. Wang GG, Allis CD, Chi P. Chromatin remodeling and cancer, Part II: ATP-dependent chromatin remodeling. Trends Mol Med. 2007;13(9):373-80.

20. Ho L, Crabtree GR. Chromatin remodelling during development. Nature. 2010;463(7280):474-84.

21. Sandoval GJ, Pulice JL, Pakula H, Schenone M, Takeda DY, Pop M, Boulay G, Williamson KE, McBride MJ, Pan J, et al. Binding of TMPRSS2-ERG to BAF chromatin remodeling complexes mediates prostate oncogenesis. Mol Cell. 2018;71(4):554-566.e7.

22. Wang Y, Wong RH, Tang T, Hudak CS, Yang D, Duncan RE, Sul HS. Phosphorylation and recruitment of BAF60c in chromatin remodeling for lipogenesis in response to insulin. Mol Cell. 2013;49(2):28397.

23. Miyake N, Tsurusaki Y, Matsumoto N. Numerous BAF complex genes are mutated in Coffin-Siris syndrome. Am J Med Genet C Semin Med Genet. 2014;166C(3):257-61.

24. Lempiäinen JK, Niskanen EA, Vuoti KM, Lampinen RE, Göös $H$, Varjosalo M, Palvimo JJ. Agonistspecific protein interactomes of glucocorticoid and androgen receptor as revealed by proximity mapping. Mol Cell Proteomics. 2017;16(8):1462-1474.

25. Li M, Zhao H, Zhao SG, Wei DM, Zhao YR, Huang T, Muhammad T, Yan L, Gao F, Li L, et al. The HMGA2-IMP2 Pathway Promotes Granulosa Cell Proliferation in Polycystic Ovary Syndrome. J Clin Endocrinol Metab. 2019;104(4):1049-1059.

26. Liu Z, Yu Y, Huang Z, Kong Y, Hu X, Xiao W, Quan J, Fan X. circRNA-5692 inhibits the progression of hepatocellular carcinoma by sponging miR-328-5p to enhance DAB2IP expression. Cell Death Dis. 2019;10(12):900.

27. Wu H, Wu S, Zhu Y, Ye M, Shen J, Liu Y, Zhang Y, Bu S. Hsa_circRNA_0054633 is highly expressed in gestational diabetes mellitus and closely related to glycosylation index. Clin Epigenetics. 2019;11(1):22.

28. Jia C, Wang S, Yin C, Liu L, Zhou L, Ma Y. Loss of hsa_circ_0118530 inhibits human granulosa-like tumor cell line KGN cell injury by sponging miR-136. Gene. 2020;744:144591.

29. Wu G, Xia J, Yang Z, Chen Y, Jiang W, Yin T, Yang J. CircASPH promotes KGN cells proliferation through miR-375/MAP2K6 axis in Polycystic Ovary Syndrome. J Cell Mol Med. 2020; DOI: $10.1111 /$ jcmm.16231. 
30. Xiao S, Chang RM, Yang MY, Lei X, Liu X, Gao WB, Xiao JL, Yang LY. Actin-like 6A predicts poor prognosis of hepatocellular carcinoma and promotes metastasis and epithelial-mesenchymal transition. Hepatology. 2016;63(4):1256-71.

31. Bao X, Tang J, Lopez-Pajares V, Tao S, Qu K, Crabtree GR, Khavari PA. ACTL6a enforces the epidermal progenitor state by suppressing SWI/SNF-dependent induction of KLF4. Cell Stem Cell. 2013;12(2):193-203.

32. Zhang J, Zhang J, Wei Y, Li Q, Wang Q. ACTL6A regulates follicle-stimulating hormone-driven glycolysis in ovarian cancer cells via PGK1. Cell Death Dis. 2019;10(11):811.

33. Chen LL, Yang L. Regulation of circRNA biogenesis. RNA Biol. 2015;12(4):381-388.

34. Li X, Yang L, Chen LL. The Biogenesis, Functions, and Challenges of Circular RNAs. Mol Cell. 2018;71(3):428-442.

35. Chen N, Zhao G, Yan X, Lv Z, Yin H, Zhang S, Song W, Li X, Li L, Du Z, et al. A novel FLI1 exonic circular RNA promotes metastasis in breast cancer by coordinately regulating TET1 and DNMT1. Genome Biol. 2018;19(1):218.

36. Good CR, Panjarian S, Kelly AD, Madzo J, Patel B, Jelinek J, Issa JJ. TET1-Mediated Hypomethylation Activates Oncogenic Signaling in Triple-Negative Breast Cancer. Cancer Res. 2018 Aug 1;78(15):4126-4137.

37. Pamudurti NR, Bartok O, Jens M, Ashwal-Fluss R, Stottmeister C, Ruhe L, Hanan M, Wyler E, PerezHernandez D, Ramberger E, et al. Translation of circRNAs. Mol Cell. 2017;66(1):9-21.e7.

38. van Heesch S, Witte F, Schneider-Lunitz V, Schulz JF, Adami E, Faber AB, Kirchner M, Maatz H, Blachut S, Sandmann CL, et al. The Translational Landscape of the Human Heart. Cell. 2019 Jun 27;178(1):242-260.e29.

39. Dong W, Dai ZH, Liu FC, Guo XG, Ge CM, Ding J, Liu H, Yang F. The RNA-binding protein RBM3 promotes cell proliferation in hepatocellular carcinoma by regulating circular RNA SCD-circRNA 2 production. EBioMedicine. 2019;45:155-167.

\section{Table}


Table

\begin{tabular}{|llll|}
\hline Parameters & Control & PCOS & P values \\
\hline Age (years) & $29 \pm 2.67$ & $29 \pm 3.85$ & 0.4703 \\
\hline BMI $\left(\mathrm{kg} / \mathrm{m}^{2}\right)$ & $21.19 \pm 2.21$ & $26.73 \pm 3.54$ & $<0.01^{*}$ \\
\hline Basal FSH(IU/L) & $6.73 \pm 1.47$ & $5.27 \pm 1.06$ & $<0.01$ \\
\hline Basal LH(IU/L) & $5.36 \pm 1.12$ & $9.13 \pm 3.48$ & $<0.01$ \\
\hline Basal T(ng/dL) & $23.42 \pm 13.27$ & $38.61 \pm 18.52$ & $<0.01$ \\
\hline AMH $(\mathrm{ng} / \mathrm{ml})$ & $6.24 \pm 2.1$ & $8.53 \pm 3.5$ & $<0.01$ \\
\hline Fasting glucose $(\mathrm{mmol} / \mathrm{L})$ & $4.58 \pm 0.63$ & $5.64 \pm 0.87$ & $<0.01$ \\
\hline Fasting insulin (mIU/L) & $8.95 \pm 3.69$ & $23.78 \pm 13.45$ & $<0.01$ \\
\hline HOMA-IR & $1.56 \pm 0.32$ & $4.58 \pm 3.12$ & $<0.01$ \\
\hline
\end{tabular}

\section{Figures}
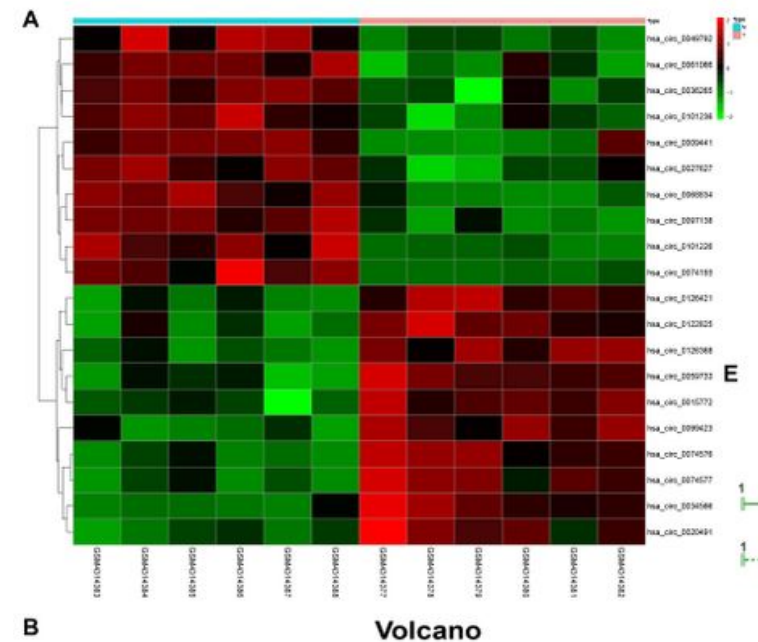

B

\section{Volcano}
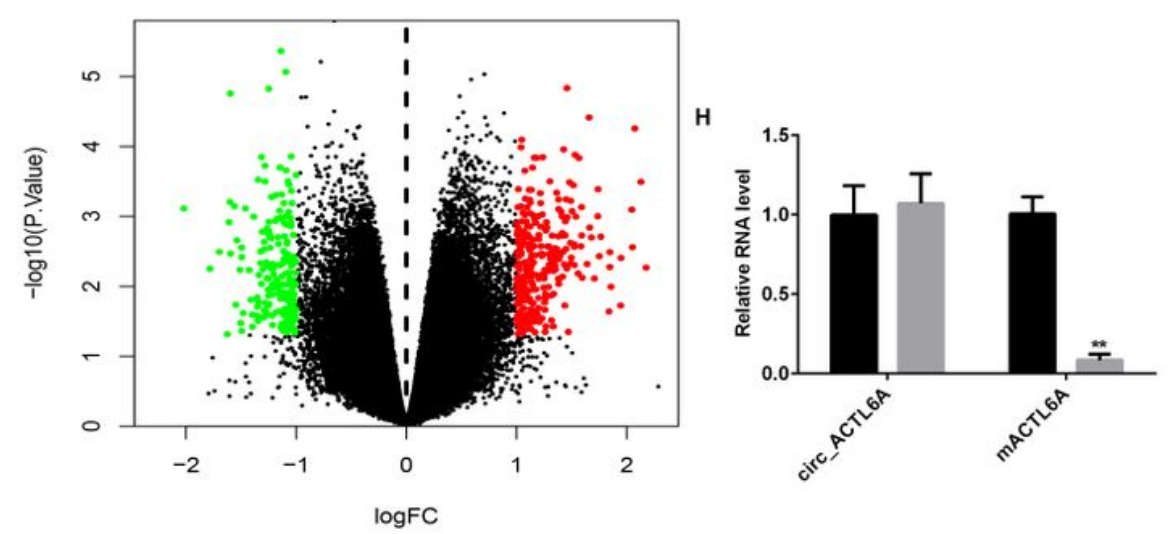
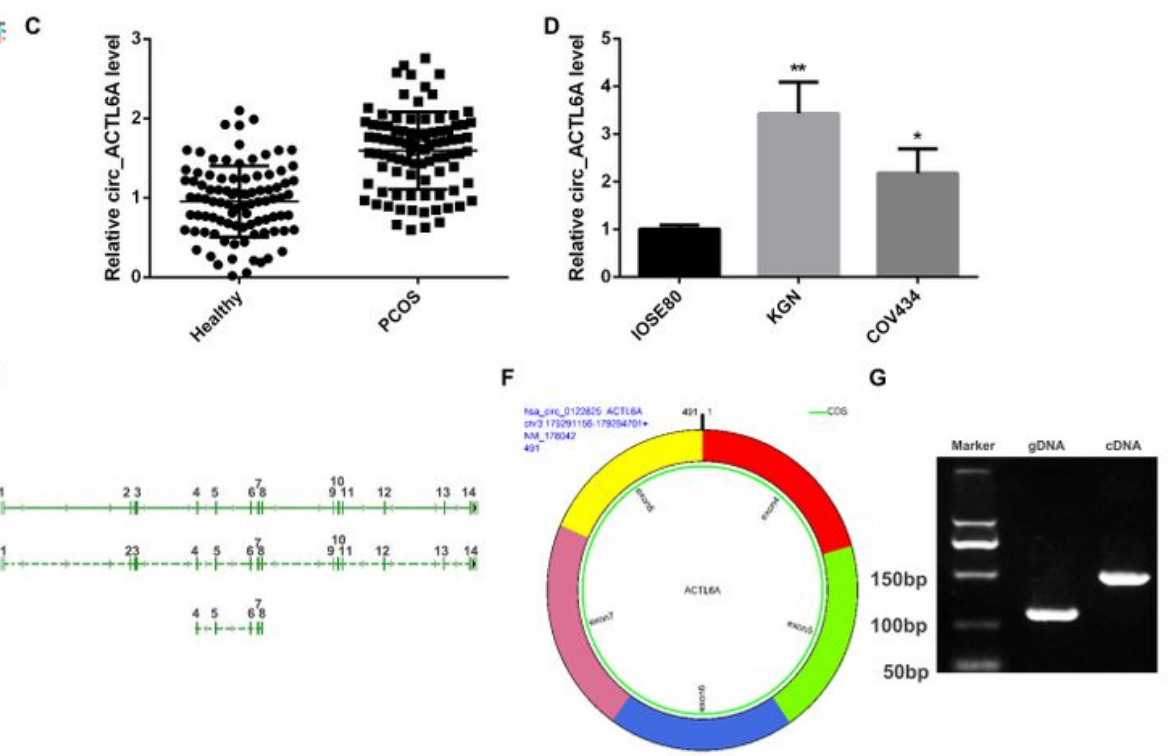

- Mock

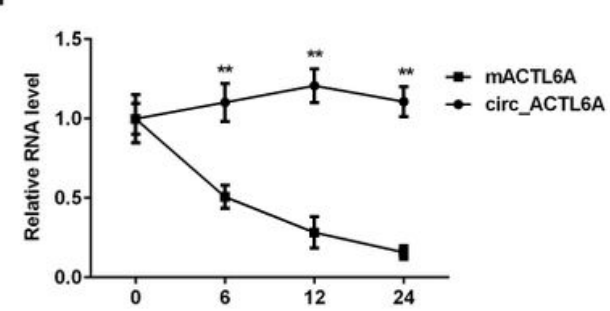

Figure 1 
circ_ACTL6A is overexpressed in PCOS A: Hierarchical clustering analysis of circRNAs PCOS patients and healthy control. B: Volcano plot analysis of circRNAs PCOS patients and healthy control. C: The level of circ_ACTL6A in PCOS patients. D: The level of circ_ACTL6A in vivo. E: Pre-ACTL6A is the parental gene of circ_ACTL6A, and ACTL6A mRNA is the mature linear RNA. F: The structure of circ_ACTL6A analyzed by software circPrimer (v.1.2.0.5). G: Divergent and convergent primers was designed to amplify circ_ACTL6A and linear ACTL6A, respectively. H: PCR verification of circ_ACTL6A. I: qRT-PCR analysis circ_ACTL6A RNA stability. **P<0.01.

A

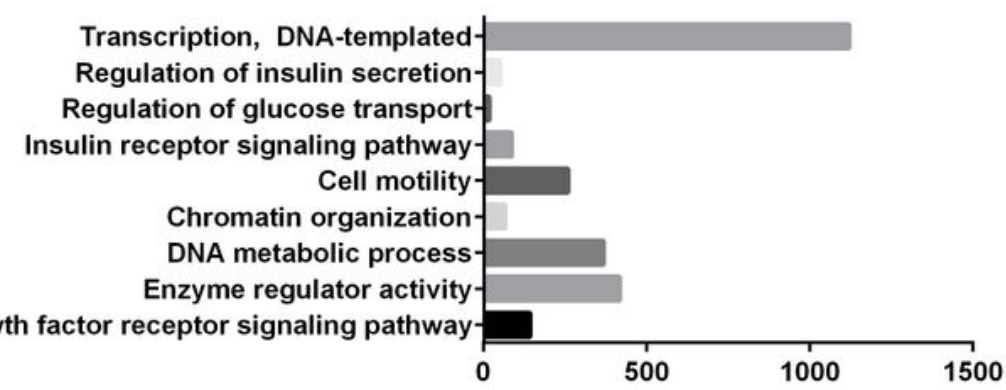

B

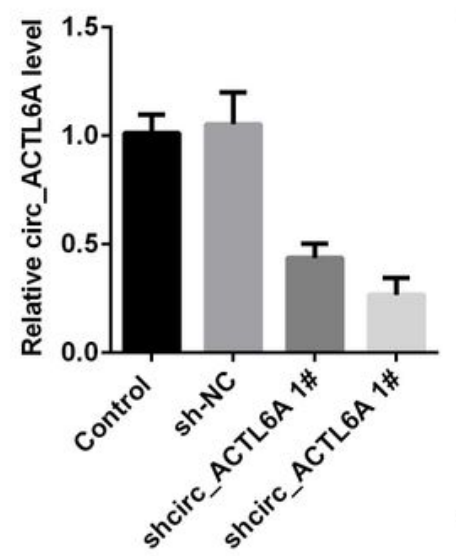

shNC shcirc_ACTL6A

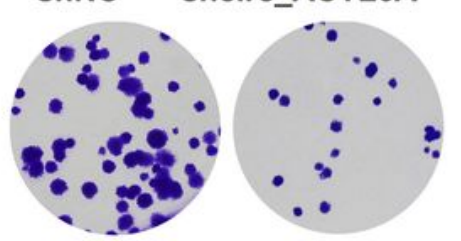

$\mathbf{F}$

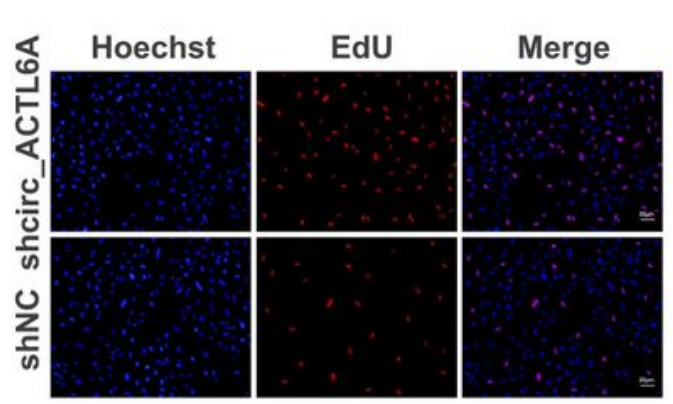

C

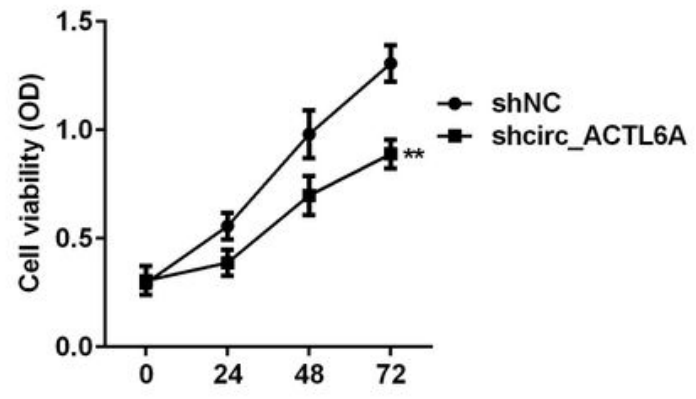

E

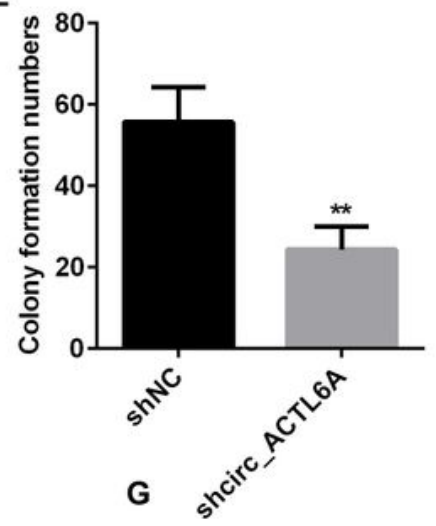

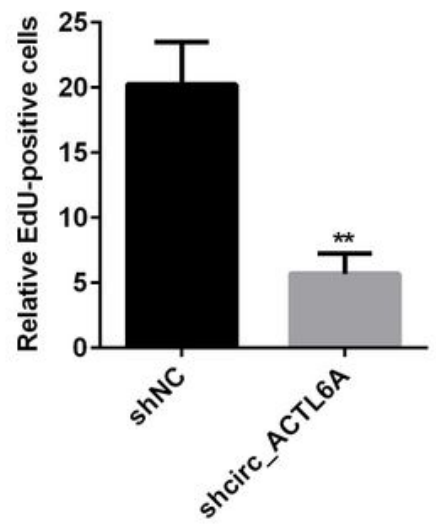


Figure 2

Knockdown of circ_ACTL6A suppressed the proliferation, migration, and invasion of KGN cells A:

Transfection efficiency of circ_ACTL6A detected by qRT-PCR. B: Cell viability determined by CCK-8. C: The colony formation measured by colony formation assay. D: Quantification of $\mathrm{C}$. E: The proliferation of KGN cells determined by EdU assay. F: Quantification of E. ${ }^{*} \mathrm{P}<0.01, \# \# \mathrm{P}<0.01$.
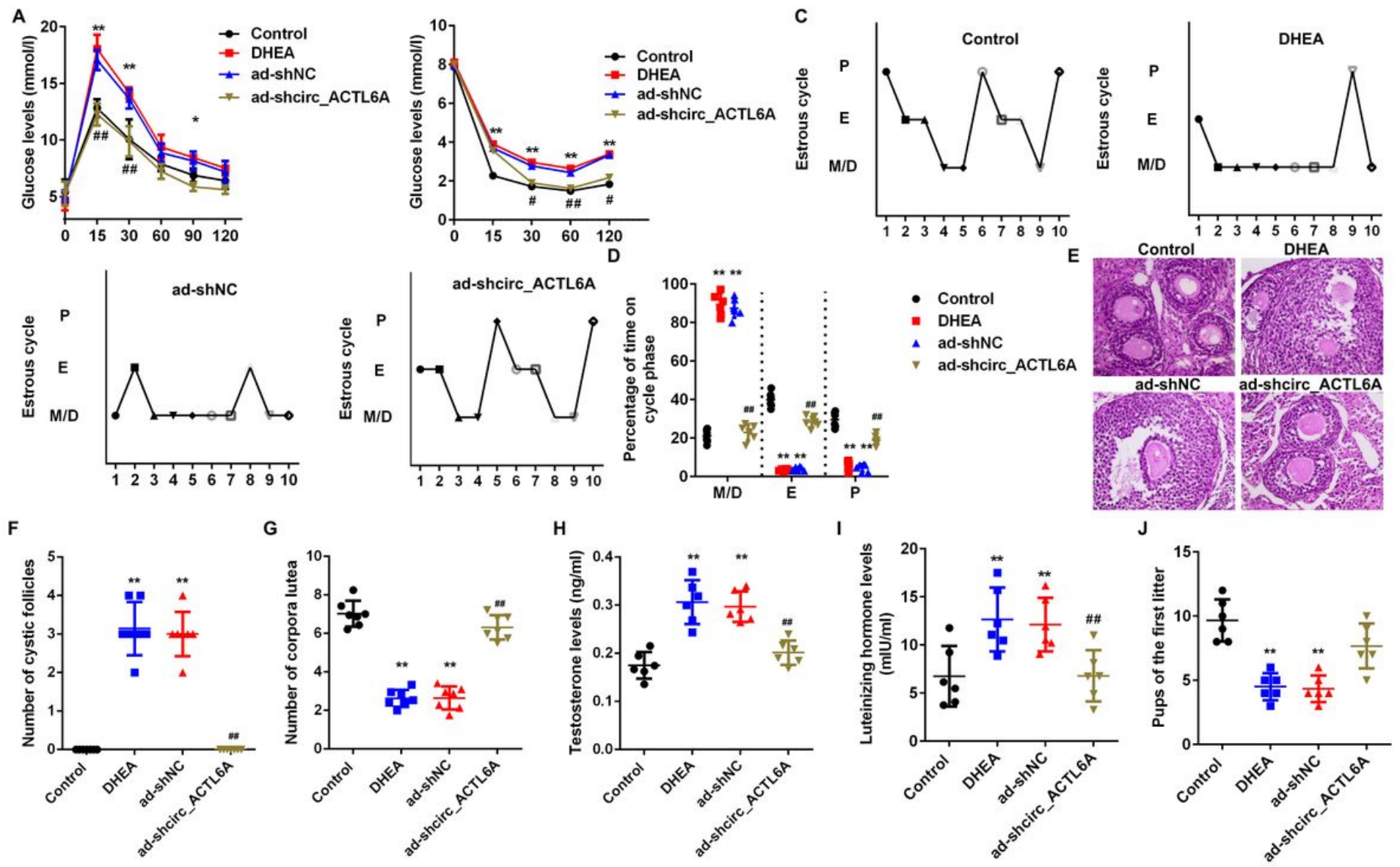

Figure 3

Knockdown of circ_ACTL6A antagonizes insulin resistance, ovarian dysfunction and infertility in mice with PCOS A: Insulin tolerance detected by glucose tolerance tests. B: Insulin tolerance determined by insulin tolerance tests. C: Representative estrous cycles of control group. D: Representative estrous cycles of mice PCOS. E: Histological analysis of ovaries. F: The number of cystic follicles. G: Quantification of corpora lutea. $\mathrm{H}$ : Testosterone levels determined by with liquid chromatography-mass spectrometry. I: Luteinizing hormone measured by an autoanalyzer. J: The fertility assay. ${ }^{\star} \mathrm{P}<0.01, \# \# \mathrm{P}<0.01$. 

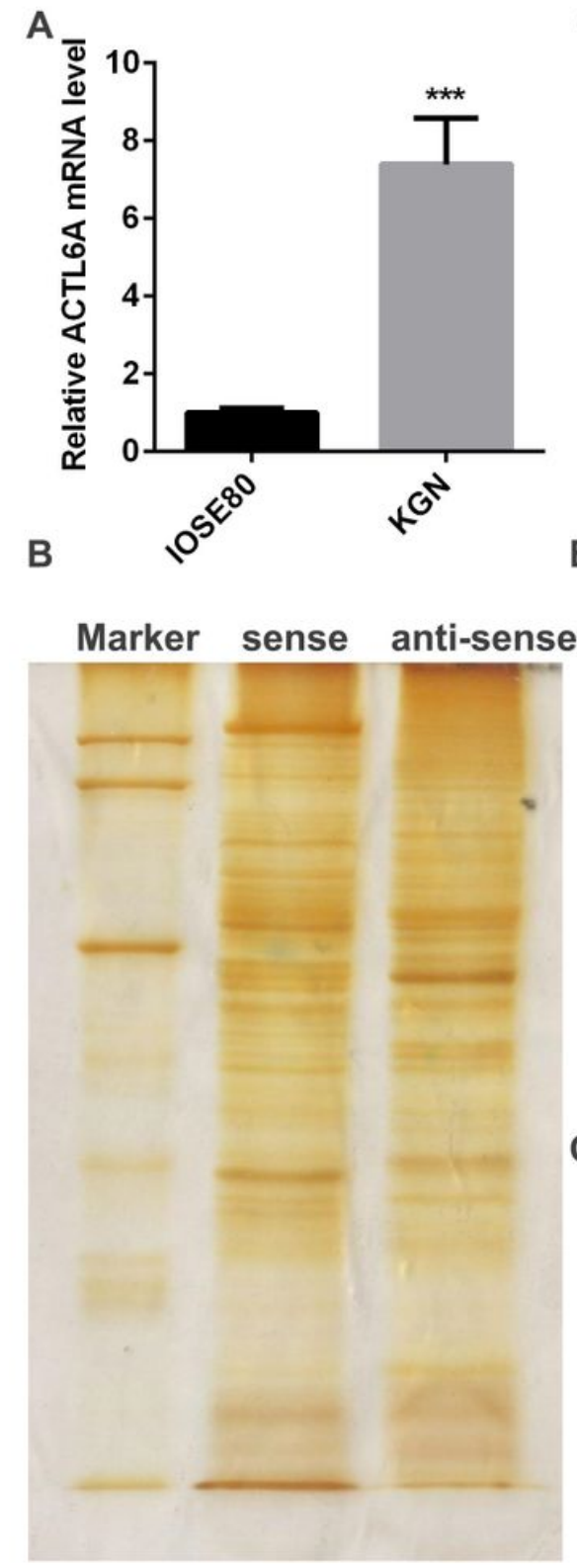

C

E

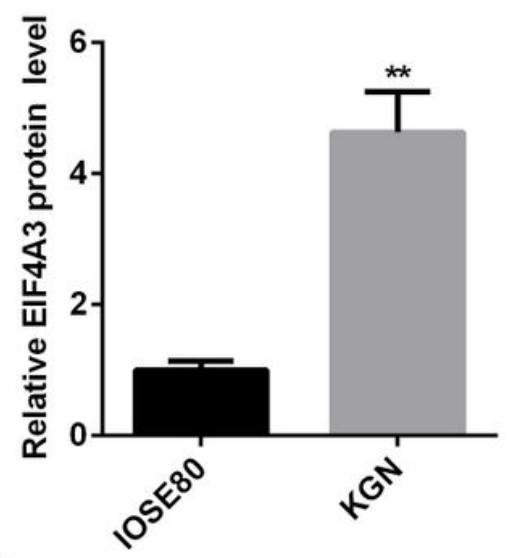

G

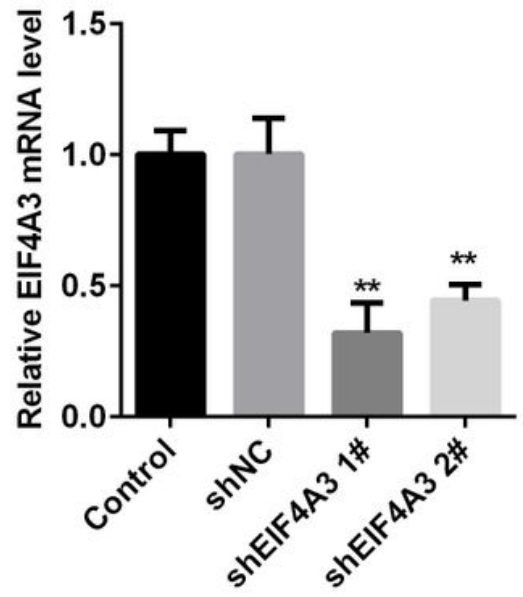

D

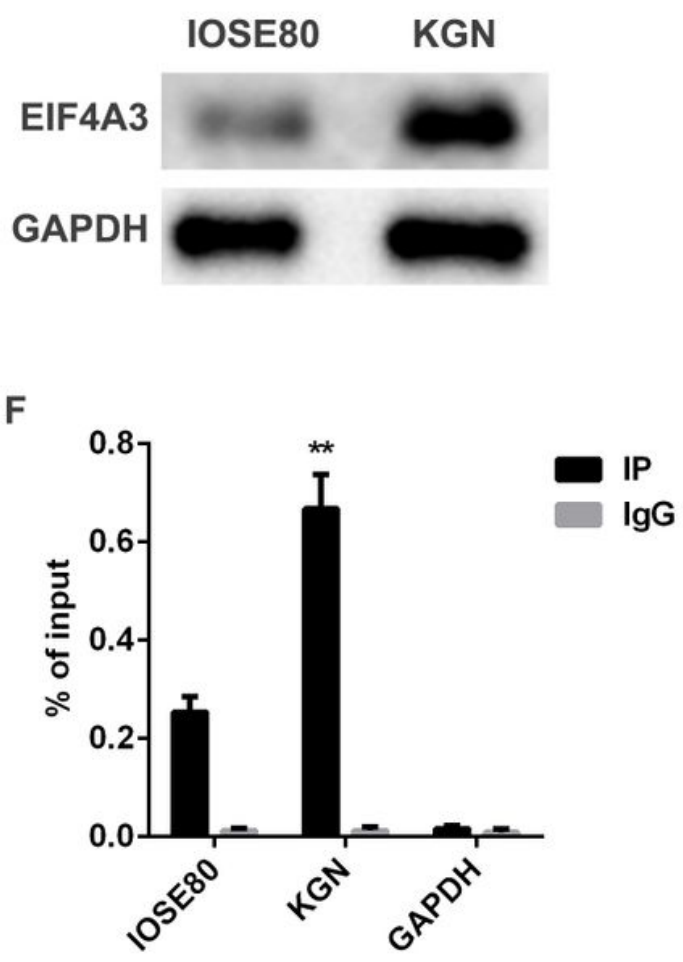

H

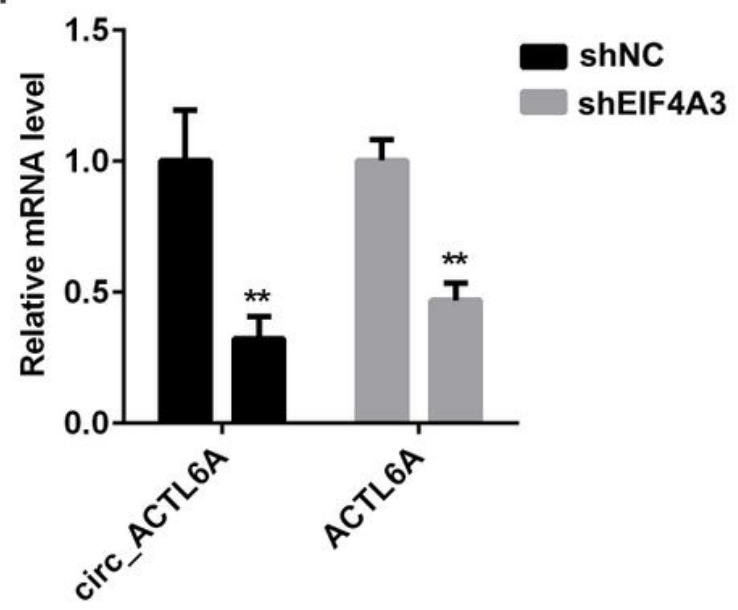

Figure 4

EIF4A3 induces the upregulation of circ_ACTL6A A: The mRNA level of ACTL6A determined by qRT-PCR. B: The protein interacting with pre-ACTL6A detected using RNA pull-down assay. C: The mRNA level of EIF4A3. D: The protein level of EIF4A3 determined by western blot. E: Quantification of D. F: The interaction between EIF4A3 and circ_ACTL6A determined using RIP assay. G: The mRNA level of EIF4A3 determined by qRT-PCR. H: The expression level of circ_ACTL6A and ACTL6A determined by qRT-PCR. $\star * P<0.01$. 

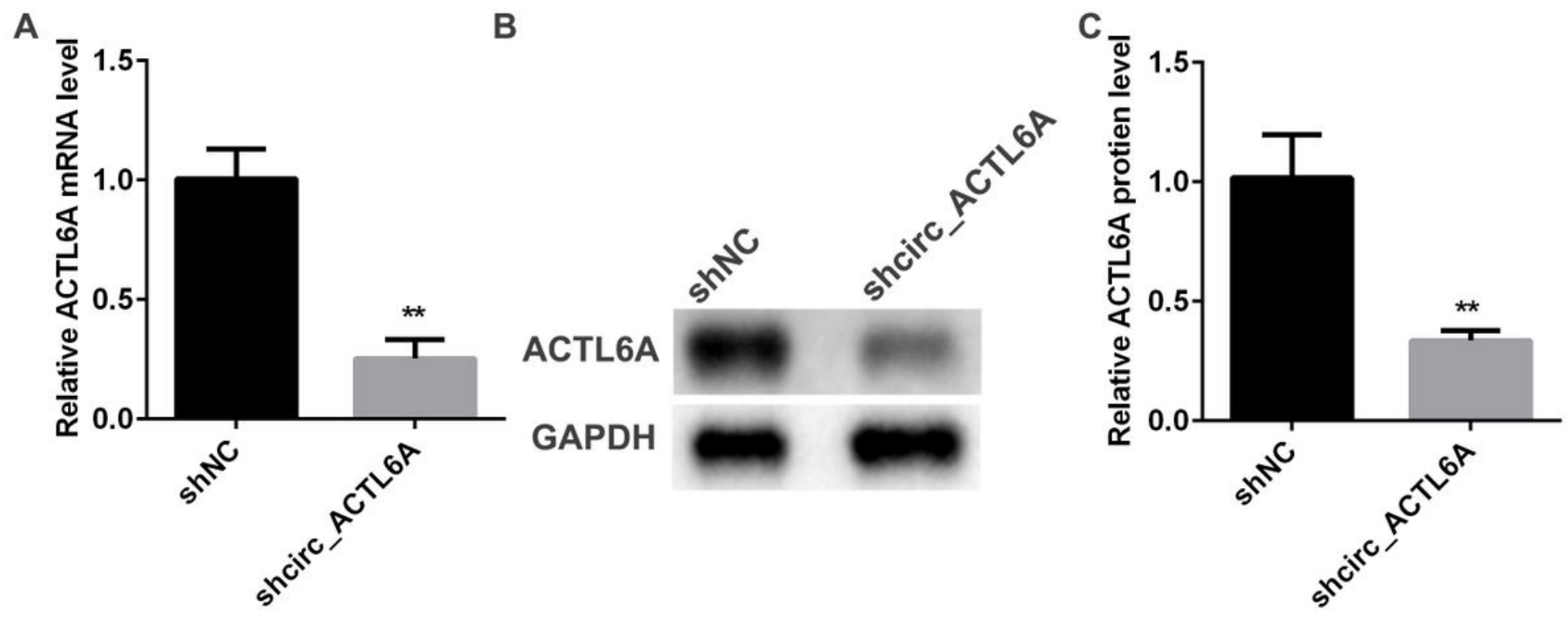

Figure 5

circ_ACTL6A upregulates ACTL6A in vivo and in vitro A: The mRNA level of ACTL6A in patients with PCOS determined using qRT-PCR. B: The mRNA level of ACTL6A in KGN cells determined using qRT-PCR. C: The protein level of ACTL6A in KGN cells determined using western blot. D: Quantification of C. E: The interaction between circ_ACTL6A and ACTL6A determined by RIP. F: The mRNA level of ACTL6A in vivo. G: The protein level of ACTL6A in vivo. H: Quantification of G. ${ }^{*} \mathrm{P}<0.01, \# \# \mathrm{P}<0.01$. 
A

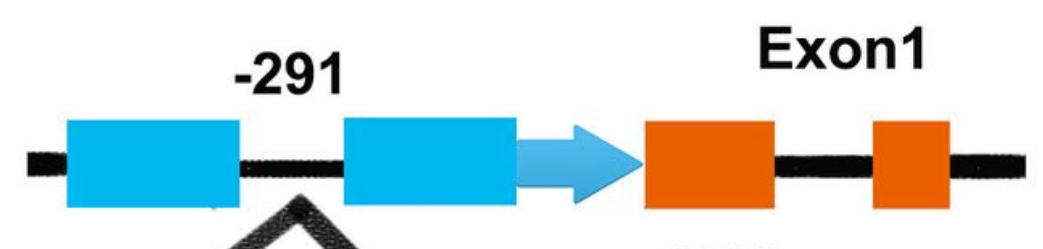

$\begin{array}{cl}\text { Tail } & \text { BstB1 } \\ \text { (ACGT) } & \text { (ACGT) }\end{array}$

B

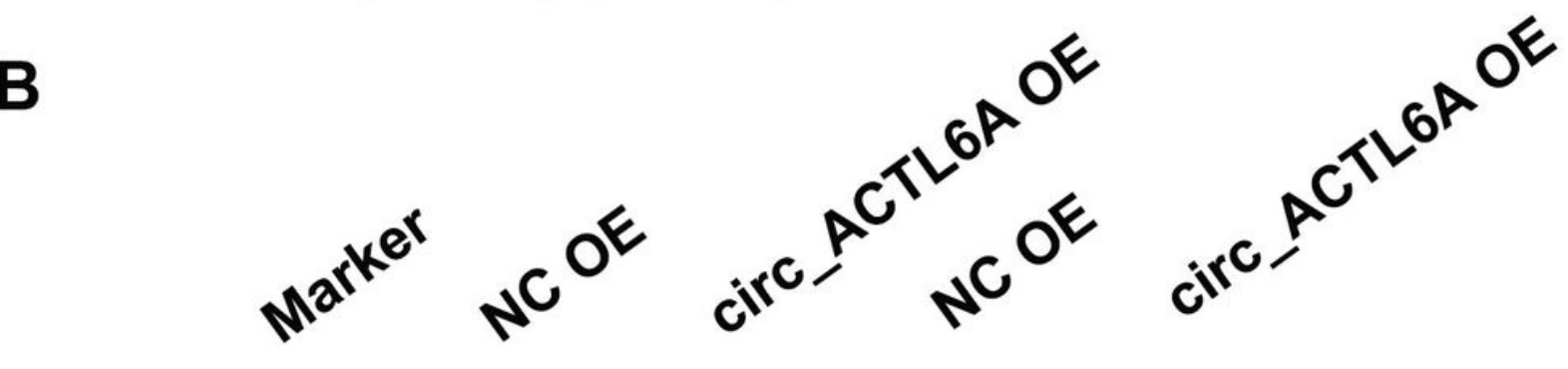

300bp--

100bp--

\section{ATG}

Figure 6

circ_ACTL6A induces CpG DNA demethylation in the ACTL6A promoter A: CpG islands in the promoter of ACTL6A. B: DNA demethylation analyzed by sodium bisulfite sequencing.

A

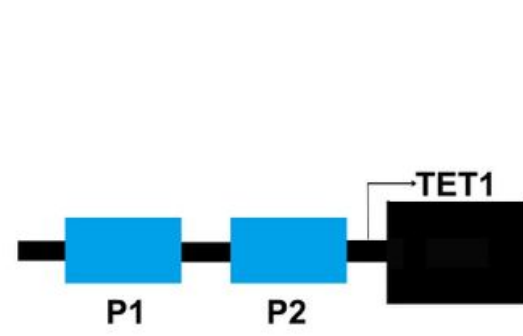

B

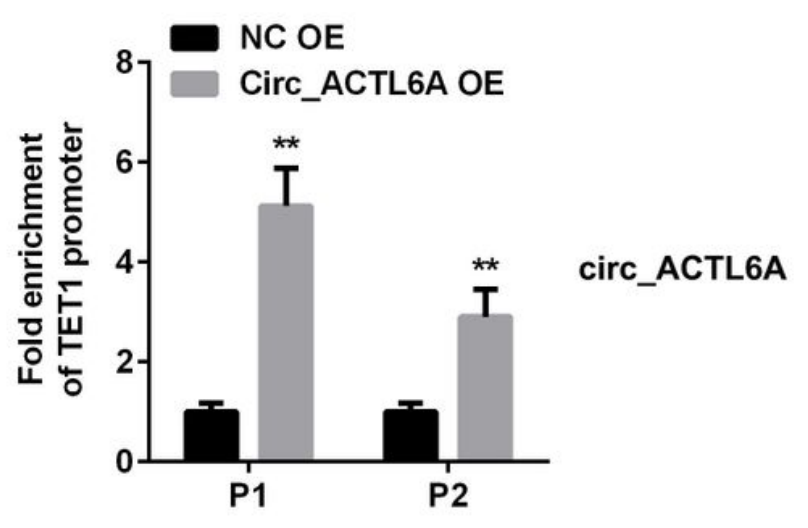

--Unmethylated --Methylated

Figure 7 
circ_ACTL6A recruits TET1 A: The expression of TET1 in KGN cells determined by qRT-PCR. B: The interaction between TET1 and circ_ACTL6A verified by RIP assay. **P<0.01, \#\#P<0.01.

A

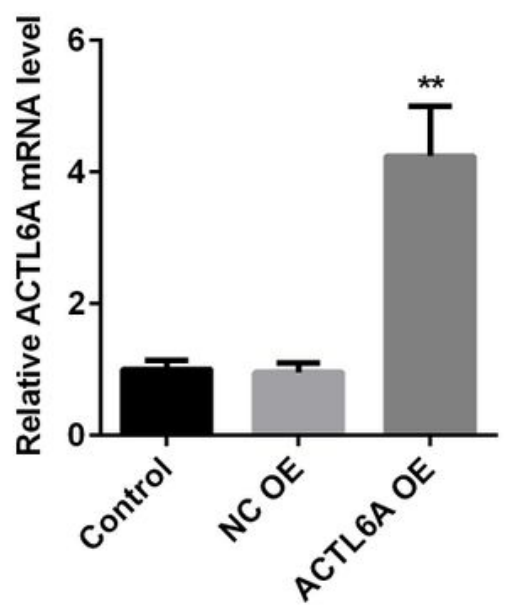

B

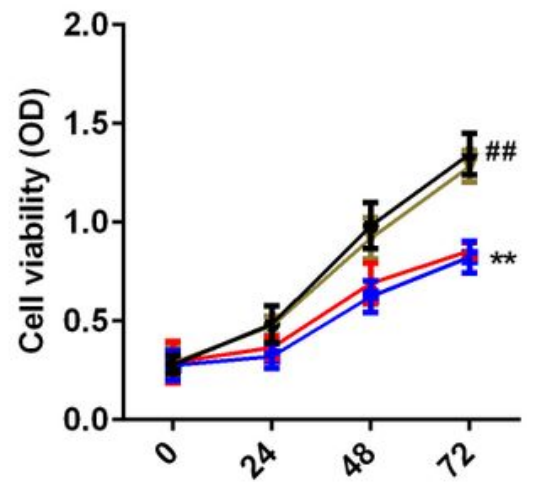

$\rightarrow$ shNC

- shcirc_ACTL6A

- shcirc_ACTL6A+NC OE

$\rightarrow$ shcirc_ACTL6A+ACTL6A OE

C
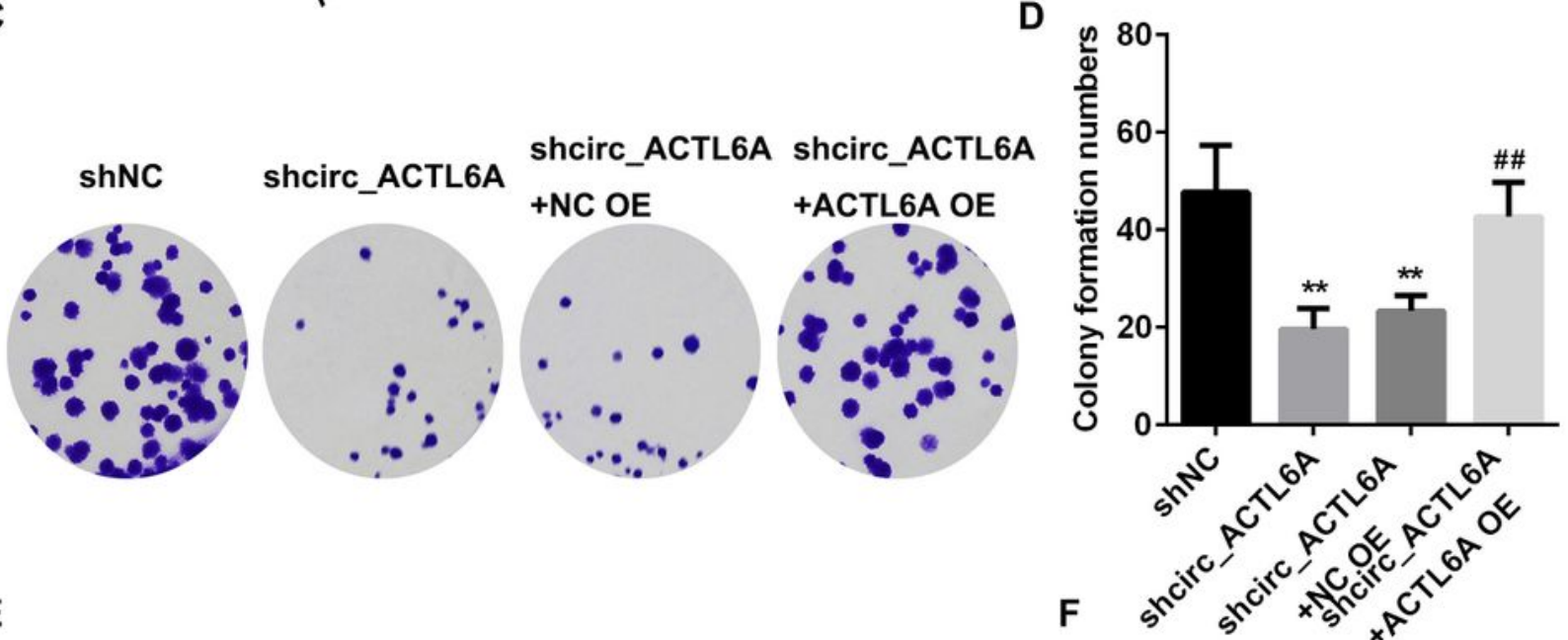

E shNC shcirc_ACTL6A

shcirc_ACTL6A shcirc_ACTL6A
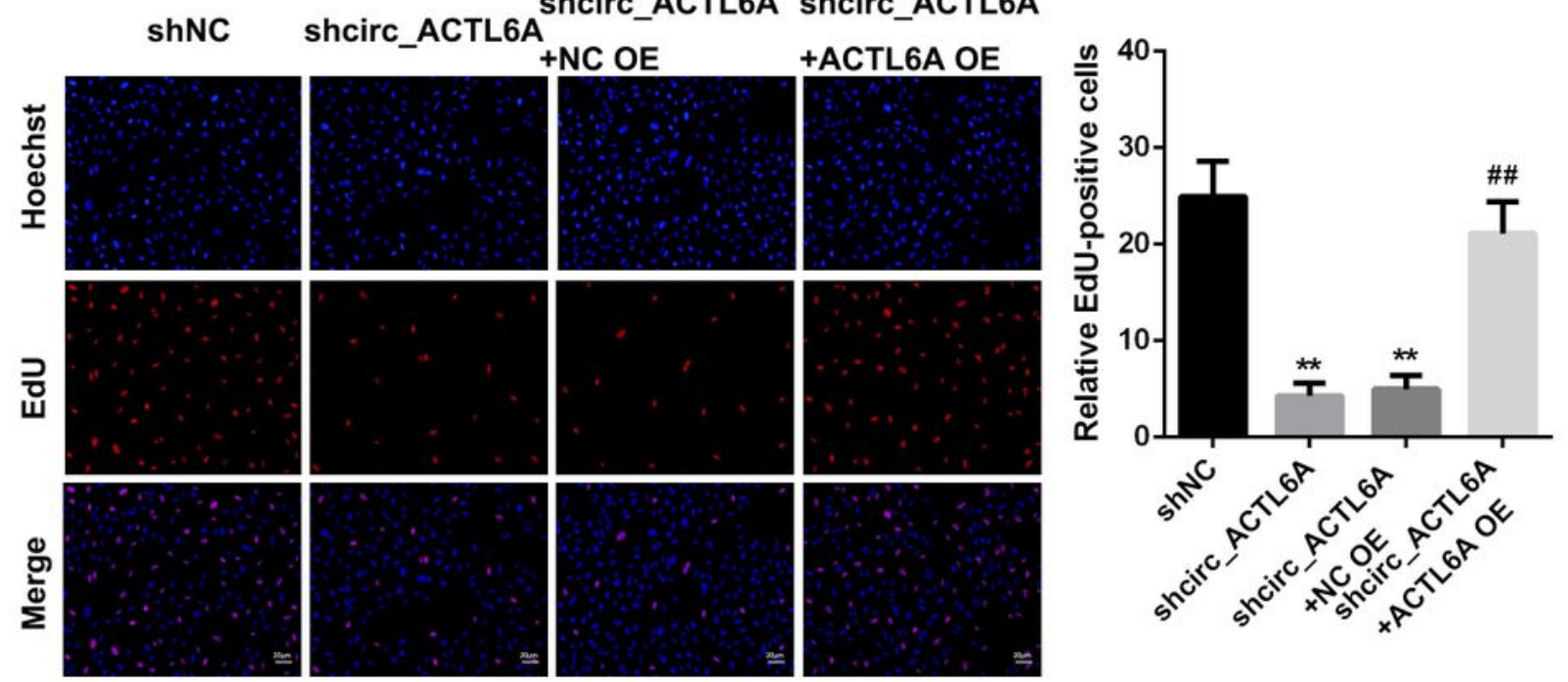

Figure 8

Overexpressed ACTL6A reverses the effects of circ_ACTL6A knockdown on the proliferation of KGN in vitro A: The mRNA level of ACTL6A detected by qRT-PCR. B: Cell viability detected by CCK-8 assay. C: 
Colony formation detected by colony formation assay. D: Quantification of C. E: The proliferation detected by EdU assay. F: Quantification of $E .{ }^{\star *} P<0.01$, \#\#P<0.01, \&\&P<0.01.

\section{Supplementary Files}

This is a list of supplementary files associated with this preprint. Click to download.

- Supplementarymaterials.csv 